Home as a hybrid centre of medication practice

\begin{tabular}{|r|l|}
\hline Journal: & Sociology of Health and Illness \\
\hline Manuscript ID: & SHI-00097-2012.R2 \\
\hline Manuscript Type: & Original Article \\
\hline Key Words: & households, medication practices, Latour, de Certeau \\
\hline \multicolumn{2}{|}{} \\
\hline
\end{tabular}

SCHOLARONE ${ }^{\text {m }}$

Manuscripts 


\title{
Home as a hybrid centre of medication practice
}

\begin{abstract}
This paper presents research that explores how medications are understood and used by people in everyday life. An intensive process of data collection from 55 households was used in this research, which included photo-elicitation interviews and diary-elicitation interviews. It is argued that households are at the very centre of complex networks of therapeutic advice and practice and can usefully be seen as hybrid centres of medication practice, where the plethora of available medications are assimilated and different forms of knowledge and expertise are made sense of. Dominant therapeutic frameworks are tactically manipulated in households in order for medication practices to align with the understandings, resources and practicalities of households. Understanding the home as a centre of medication practice de-centralises the role of health advisors (whether mainstream or alternative) in wellness practices.
\end{abstract}

Keywords: households; medication practices; Latour; de Certeau

\section{Introduction}

This paper develops the argument that homes can usefully be portrayed as hybrid centres of medication and therapeutic practices This signals the ways in which the household cuts across primary and secondary care, mainstream and alternative practices, and links into a wide array of networks - including family, friends, libraries, the Internet and the whole range of health advisors. The breadth and depth of connections and activities are assimilated in the home and the form of the assimilation is influenced by internal household relationships.

A fertile way of exploring the household as a centre of therapeutic practice is in relation to Latour's notion of hybrids. Latour makes the argument that what he calls "the moderns - are a type of people who maintain a belief in the existence of pure categories, seen in the headings of daily newspapers: "Economy, Politics, Science, Books, Culture, Religion and Local Events" but at the same time "all of culture and all of nature get churned up again every day" (Latour 1993: 2) in the form of "unruly hybrids" (Blok and Jensen 2011: 55). Households can be seen as hybrids mixing up and reassembling what has been 'purified' in other health practice spaces such as in the office of the mainstream medical practitioner or the consulting rooms of the alternative healer. Although from this Latourian perspective these latter two are inevitably hybridising purified categories as well, there are practical and discursive efforts to separate out various domains - such as science from non-science, orthodox from unorthodox, natural from artificial or synthetic. But these get mixed up in household spaces where responses to illness and wellbeing involve pragmatic decision-making based on what seems to work or what might work rather than purified rule-following.

Households engage in their own form of 'truth production' through research, experimentation and observation. In other sites of 'truth production, such as science and the law, the truth is standardised across sites. Williams and Popay contend that 'lay beliefs' pose little direct challenge to the power of the medical profession because they "remain outside the worlds of science and politics" (Williams and Popay 1994: 118) and are disorganised. Here it is argued that lay beliefs and practices are inherently a challenge to the power of medicine, in particular because they are not readily visible and therefore not readily disciplined. There is a translation of processes where the attempts to have centralised control through centres of calculation are undermined, and these products of calculation, such as the medical prescription, the clinical 
advice and the regimens of wellbeing are re-worked and re-formed. The work of purification of scientific medical practice is hybridised in the daily activities of households enacting wellness.

For Latour a centre of calculation is able to coordinate scientific practices that are widely spread, both in terms of time and space (Latour 2005). If we apply this to medicine we can say that the centre of calculation can bind many sites to it through such activities as evidence-based practice, quality assurance and practice guidelines (Dew 2001) enacted through the various medical councils, colleges and schools. By standardising practices the centre of calculation gains control over the actions of others, even where those others are at a distance (Latour 1988; Latour 1992a). For the medical elites, power is gained by developing treatment guidelines that all practitioners can follow and that can be applied to whole categories of patients. From one "place" the medical profession can control many practices. But households decentre and unravel these binds. Homes are sites where wellness and remedy is performed - and not directly overseen (exceptions would include home visits to administer medications to the confused elderly). Homes are sites where consumer goods are appropriated and individualised (Tilley 2006). It is argued here that medications, as goods, are indeed personalised and reconfigured in the home and it is suggested that we could usefully consider the home as a centre of health care decision-making and practice.

Householders are faced with the "normative frameworks" of therapeutic practices provided in such institutional spaces as the GP clinic, pharmacy, health food store and the alternative therapist's consulting room. This research draws on de Certeau's suggestion in relation to popular culture that the discourses provided through these spaces are not just normative frameworks, but "tools manipulated by users" (de Certeau 1984: 21 - italics in original). de Certeau's concern is to analyse the "micro-subversive tactics in the practice of everyday life" (Ward 2000: 8). His analytics allows us to attend to the practices of people in relation to established rules and ideas (de Certeau 1984). People are not passive in relation to 'dominant' discourses, or externally imposed rules of operation, but actively and tactically engage with them. de Certeau's approach is to render logical "such apparently aberrant ideas as the power of the powerless, the activity of the passive, the productions of non-producers" (Buchanan 2000: 98). This production of non-producers is not seen in new products, but in the way products that are "imposed by a dominant economic order" are used (Buchanan 2000: 99). For de Certeau consumption is a form of production (de Certeau 1984). As such, this research explores the active processes of production of medication practices in a 'space' that is generally hidden from view and where medications are assumed to be passively consumed.

Householders then "make (bricolent) innumerable and infinitesimal transformations of and within the dominant cultural economy in order to adapt it to their own interests and their own rules" (de Certeau 1984: xiv). de Certeau, is interested in examining the popular procedures that "manipulate the mechanisms of discipline and conform to them only in order to evade them" (de Certeau 1984: xiv).

This paper is informed by the concepts of hybrids and tactics to illuminate the networked nature of household medication practices and the re-assembling and subversion of received frameworks. The analysis not only provides a sociological understanding of the household as a centre of medication practices but also raises important issues for health practitioners in terms of situating their own practices in relation to their patients and the limitations of seeing decision-making as a one-off occurrence made in a disciplined space.

\section{Methods}


This multi-disciplinary project used households as the sampling unit since we have little knowledge of what happens with medications once they find their way into homes. Fifty-five households were purposively sampled from four cities across New Zealand to ensure some diversity in the sample and included a variety of household compositions and ethnicities, households where chronic illness was present, households with children under twelve years, and households where either CAM or dietary supplement use was prominent. Potential households were sought via a variety of means, which included advertising, snowballing, the use of networks and approaches to support groups.

Insert table 1 about here

This sampling classification proved rather arbitrary since most households fit several of the criteria, with for example, prescription medications, alternative medications and dietary supplements being relatively common. Defining medication was also potentially problematic. We used the term very broadly, to include anything taken for therapeutic reasons - to treat, cure, or prevent symptoms and illness, and to sustain health. Hence we sought discussion about anything that participants understood as medication-like, covering prescription and over-thecounter pharmaceuticals, dietary supplements, alternative medications and elixirs. We specifically excluded illicit drugs from consideration.

Data collection involved a range of methods, including mapping the home and locating all medications, photographing those locations, asking participants to produce all medications and discuss them as a household group, keeping a medication use diary, keeping a diary reflecting on medications in everyday life, and completing a photo-elicitation project to show the world of medications. The diaries and photographs were then used as the basis for further interviews where the diary entries and photographs acted as prompts for the participants. These methods were chosen to add richness and depth to the data. In particular, mapping and producing all medications in the household extended discussions as the materiality of the products demanded remembering and accounting, for why they were there, what they meant, and how they were used.

For the analysis reported here, the first author read household interviews to identify the kinds of therapeutic practices deployed in households. The theme of this paper arose as an outcome of this process and other team members were involved in workshopping the ideas at a writing retreat. This analysis was then revised and developed by the other authors in an iterative process. This iterative analytic work developed a number of theoretical ideas and interpretations from the data, focussing around the practices of household members in relation to their use and understanding of medications. These ideas were discussed and refined around the major theoretical focus of this paper, the household as a hybrid centre of medication practices. For this paper medication practices identified in the households have been organised into two sections: 1) diagnosing and prescribing 2) sources of practices. Ethical approval for the project was obtained through an institutional ethics committee.

\section{Diagnosing and prescribing}

Diagnosis and prescription might be considered to be the preserve of clinicians and Prior argues that patients are not skilled or practised in diagnosis (Prior 2003), but we find that these activities are ubiquitous in households. In developing a treatment plan householders observe, experiment, assess, draw on a range of advice and self-prescribe. Householders deviate from or 
reject advice and develop their own expertise. A critical dimension of diagnostic activity is to determine when outside help is required. As Jutel notes, before any encounter with a health professional a patient makes a premedical assessment (Jutel 2011). Close observation and identification of the 'normal' are central elements in determining severity of conditions. This can be for minor conditions, seen in one of our respondents (Natasha) when describing her decision not to take her daughter to a doctor as "she's not, you know, green mucous or anything so probably no infection”. It can be for more severe conditions, seen in another respondent's (Hazel) decision not to take her fevered child to the GP, where there have been past concerns about febrile seizures, "because I know that that's the way she gets sick". Householders combine their own medical understandings and past experience with observations to make diagnoses, prognoses and treatment plans in the home. This vigilance aligns with Williams' view of mothers as alert assistants in relation to children with chronic illness, where the needs of the children are identified and anticipated (Williams 2000). In being alert assistants householders can be acutely sensitive to the nature of each other's condition.

Professional expertise was not usually a first resort and self-prescribing was a feature of home wellness practices. Fleur's son Jason stated that “we don't go seeking help straight away”. He ridicules professional advice: "when I know I've got a sore throat I don't want to go and pay somebody to tell me I've got a sore throat". Trystan, his brother, had been given a diagnosis of gout by his doctor but the doctor just wanted to keep doing blood tests, so Trystan decided to self-prescribe, which entailed modifying his diet. Many households use what could be termed 'folk' remedies. When asked if there were any household remedies used for "sore throats and things" Jessica's household collectively listed lemon, honey and ginger tea, salt and water and baking soda for "upset stomachs and things like that". Recommended prescriptions from outside the household could be supplemented with others, such as antibiotics with selfprescribed probiotics or high doses of vitamin C.

Householders commonly deviated from the recommendations of others - whether orthodox or unorthodox, tactically engaging with them and adapting them to their own interests and rules (de Certeau 1984). Deviations included stopping medications, rejecting medications and ignoring warnings. Dan justified having an unfinished packet of antibiotics to his wife by saying "it was probably a case of if I forgot to take it for a day and then there was no point in trying to just restart the course”. Dan draws on his own understandings of how antibiotics work as a defence. Mary, an older woman, and Jim, her adult son, both rejected recommendations to take antibiotics as for Mary "You don't need them". Dave takes rum with Lemsip even though the packet states "Danger: you're not supposed to have with alcohol". He justifies this "because I don't think it's very dangerous. I think it's just kind of a lemon drink". Dave also resists his doctor's advice to take blood pressure pills, which he keeps in the bathroom, because he does not think he needs them "so as a result of that I kind of forget" to take them. Tania was concerned about the adverse effects from the GP-prescribed blood-thinning medication Warfarin for "clots on my lungs" so she "stopped taking it". Tania also stopped taking a prescribed diuretic as "It's better to just drink water and that flushes it through and have parsley tea and stuff - natural diuretics".

Expense would play a part in variations from recommendations. Paula keeps fish oil in her kitchen cupboard and has been taking it for 19 years and said "if you look at the label you're supposed to take three a day but I've only ever taken one. Too expensive to take three”. Decisions about whether or not to take a drug could be based on the impacts of its effects on activities. Paula and her husband have a pill container they put out at breakfast time. She did not take a prescribed diuretic from this container one day as she was going out and "being a 
diuretic you have to keep running to the loo all the time". She had not disclosed to her GP that this was how she managed the situation, suggesting that "I don't think...the odd day hurts not to take it because the next day I will take it”.

Janice rejects a candidate diagnosis from an osteopath that she had schleroderma as she googled the term and found that she had "less than five percent of the symptoms". Ingrid takes a prescribed sleeping pill but she does not tell her Ayurvedic practitioner "because he'd likely have a heart attack! He can't believe that somebody would have a problem with not sleeping". In this instance Ingrid is deviating from the recommendations of an alternative practitioner to follow more conventional advice. By contrast Janice provides an account of having bowel problems and her doctor suggesting "Basically don't eat anything that's going to stimulate your bowel and then just do it manually every day" by using an enema. Janice did not pursue this advice and instead followed the advice of her naturopath to take an appetite stimulant, which she keeps in the pantry. Janice also notes that in reference to another prescribed medication "as you can see I didn't take much of it. I just hate taking things that I can't really understand the purpose for". So we see Janice making decisions in the home to reject medications even after collecting the suggested medication from the pharmacist. Paula reduced the sleeping pill she was prescribed to "a quarter" of one pill. She stated that "I reduced that myself. I'm just getting myself off it - I don't want to become addicted”. Further, because of getting bad stomach pains from taking a medication to prevent osteoporosis Paula has "sort of put that on hold in the meantime", a decision she made by herself without discussion with her GP. In Tania's case we see the complex issues arising from a medication to prevent acid reflux "which basically what it does it is stops the ...production of acid but then if you don't have acid it doesn't digest your food" so she reduces the prescribed dose as "I get a problem with processing protein”. Householders hybridise wellness practices - taking some suggestions from different places and recombining them in relation to their own understandings.

Decisions made about expiry dates are also made in the home. Decisions could be based on the value placed on the medication, not the 'expert' advice on the bottle. For Jessica, an asthma drug used for her children kept in the household first aid kit "that you can't actually get anymore" is out of date but "it's like precious cargo" and "will still be alright". Dave contests the claims about expiry on a cough mixture: "I can't believe anything would ever be a problem with that". Zoe, who positions herself as a hoarder, has older medications packed neatly in a box: "I find it hard to chuck stuff out" and will use antibiotics that have "gone past the use by date" for her bad cough

Some householders had developed particular expertise in wellness practices. For example, Fleur had expertise in homeopathic diagnosis and prescribing and all family members would take homeopathics. She used different doses and prescribed for a wide range of conditions including infection, overeating and sunstroke and her son stated that "We even treat the dog". Sylvia had developed expertise in the use of essential oils and some she would "recommend to all and sundry", especially her four-year old daughter and husband, but for some of the oils she would suggest that others should consult with a professional "who knows them better than I do". Here households are sites of truth production but limitations are placed around the application of their expertise. For some the lack of expertise was a concern. Bryce had taken a photo of medications in the supermarket, noting that "at least half of one side of an aisle is just medications" and that "You can take all these things without actually knowing what they're doing to you or how they could interact with each other. All the adverse effects". 
Householders may become the expert in relation to orthodoxy. For Hazel this was a necessity as she was not able to fully trust health professionals to look out for her even though "she is allergic to some antibiotics" and "could die" if she took them again. She told the prescribing doctor:

I'm allergic to sulphur and he looked at my notes and said, 'It's not in here that you're allergic to sulphur' which made me a little bit upset because I've been going to that practice for a long time...but that's why I'm always insistent myself. I take it upon myself because I don't ... it's not that I don't trust doctors - sometimes I don't - but I just think I've got to take responsibility for that one since it's so serious.

This example illustrates a requirement for intense self-surveillance in some situations and the importance of prescribing expertise.

Householders could become experts through their own training and through necessity, but also through processes of experimentation to determine such things as the right dose for them, when to take medications and when not to. Their homes were sites of truth production. Ingrid knew that if she took too much Vitamin C "it gives you diarrhoea. That's how you know so you don't do that". Louisa was taking a homeopathic medication for urinary tract infection (UTI) "I got to three drops once a day then I started having UTIs so I did four drops once a day. Hallelujah, it seemed to work so I left it at that". Louisa did something similar to determine the right level of prescribed medication. Because "anti-depressants get a bad rap" she tried to get the dose down "and by the time I'd got down to having $20 \mathrm{mg}$ a day I was an absolute mess" and so in consultation with her doctor she increased her dose again. This experimentation from Louisa was due to her view that "I take an awful lot of medications and I want to be sure that the medications I'm taking are the medications that I need to take". Hazel stated that in general "I've actually experimented a lot with a lot of different things. I've taken lots of homeopathics, I've taken lots of Vitamin B if I felt particularly stressed or whatever. Yeah, definitely and even more extreme things and herbs and Chinese herbs". Self-observation commonly led to the rejection of particular medications. Zoe took a herbal preparation she described as a "sugar killer" for weight loss, stating that "It puts you off everything. Didn't work”. Mark rejects a remedy for his joint problems because "I tried that but I didn't notice any difference so I didn't buy anymore”. Louisa had a prescription from a homeopath "but I didn't find it helpful”. Bethany was taking a painkiller for her back injury "but I just felt it put me on a trip and I absolutely hated it". Janice rejects a prescription to have gluten free bread, as suggested by her naturopath, because through her own observations she had "come to the conclusion it's not the gluten, it's the yeast". A process of trial, error and re-trial is common in households.

Another household had a variety of cough mixtures. Natasha states that one cough mixture "is disgusting" and her daughter affirms this, but it was bought because "other cough medicines weren't working ... Just trying out different things". For whooping cough Natasha had "tried a lot of stuff”. Dan had tried "just about everything" for his hayfever and a pharmacist recommendation was not only the cheapest but the most effective. Experimentation could evolve over time. Tony took four Panadol for headaches: "I've been taking two since I was little but now that I'm older it's not as strong so I decided to see what happens with three and four and five and four was the best". Louisa could not get to sleep because "I was so wound up and uptight and tense and headachy" and found that painkillers could help "so I got in the habit of taking a couple of Paracetamol before sleep". These are placed in the bedroom for accessibility, but separated out from her husband's medications. Self-monitoring could be required to 
determine when it was best to take medications. For Louisa "I have concluded is that when I'm really tired and I take Avonex the headaches are much worse". Householders engaged in a considerable amount of such experimentation to determine what they considered as 'best practice' for themselves, rendering households as engaged sites of truth production.

Experimental concepts could be explicitly drawn on. Natasha spoke of a friend who was using a prescription medication alongside other medications. Natasha did not support this, stating that "I'm a bit of a purist...I like to take one thing so that I don't want too many confounders... If something's working then I want to know what it is". Similarly, Zoe stopped taking a menopause formula as she "decided how the hell was I ever going to know if it worked or not".

Some householders are well aware of the difficulties of determining cause from simple observation. Avril did not want to take antibiotic cream for rosacea so tried "a strange mixture of herbs". Her condition improved but she stated "I don't know if it's the change of season but my rosacea is much less bad than it was. It was all lumpy and now it's gone smooth. So whether or not it was related to that Chinese herb I don't know". Similarly she stated that after taking a homeopathic remedy for hayfever, where she was "sneezing really terribly" she subsequently "didn't have any trouble for the rest of the day. Whether or not it was that or it was just coincidence but anyway, it seemed worth it for the sugar pill or whatever it is [laughter]". Coincidence and the idea of a sugar pill (touching on the concept of placebo) are given some weight as possible explanations here. This ambivalence is further played out as Avril refers to another homeopathic remedy where she "didn't notice any difference whatsoever". So Avril self observes and notes changes but is cautious about assigning positive effects to the alternative medications she tries, though is more emphatic in noting when they do not work.

In the examples provided so far householders' views on wellness practices tend to align, but this is not always the case. Janice notes different approaches to medication in her household where her son prefers a Nurofen for a headache but Janice would prefer that he has "a drink of water first, because I think hydration is a huge thing with headaches". In relation to her sons' prescribed medications "Patrick, he's still quite blindly, 'Well, it's a doctor, they know' whereas Sam's had a few ups and downs and he's more, 'I'll tough it out'”. Janice, the mother, takes opportunities to ask questions, gauge developments and offer advice, and at the same time respond to the different orientations that her children have to medications and health professionals.

Disputes could also occur over the effects of different remedies. Sylvia suggested that an essential oil she used to bring on contractions during childbirth "worked very good", but her husband retorted with "On that point I say no, it didn't because she was still born by caesarean 10 hours later [both laughing]". In the same interview Sylvia stated that she used a particular blend of essential oils "for a pain killer for ladies' issues". This gets picked up by her husband who states that a Mirena implant resolved the severe pain, and not the oils, to which Sylvia concurs aligns. Differences of opinion about efficacy are expressed as the discussion unfolds. Households themselves are not purified, unified and categorizable sites of wellness production and medication consumption.

Diagnosis and prescription practices in the household have to occur as householders determine when and who, if anyone, needs to be consulted, how to handle untoward effects of prescribed medications and how to reconcile advice from alternative sources and with their own experiences, observations and understandings. These practices can vary from the advice 
provided by outside experts, with advice being supplemented, modified or rejected, and through this householders can challenge what is expected of them engaging in what Latour calls anti-programmatic practices (Latour 1992b). Through observation and experimentation households are sites of truth production, and by varying, rejecting and combining recommendations we can see householders drawing on norms but manipulating them to their own ends. The following section demonstrates the networked and hybrid nature of households by examining the sources for their wellness practices.

\section{Sources of Practices}

Households are embedded in extensive and sometimes overlapping networks that can span across generations, workplaces, friends, relatives and health advisors. These are sources of practices for wellness which households mix together and hybridise (Latour 1993). The term health 'advisor' is here used deliberately to illustrate the notion that clinicians and specialists feed in to household strategies but by no means dictate them. Other sources come through their own research, using libraries, the Internet and systematic or opportunistic reading.

Unsurprisingly the advice of health professionals would find its way into the home, but householders had to determine what health professional to trust. Medical doctors, although universally consulted, were not always seen as the most trustworthy. Pharmacists were frequently sought out for advice. Tania had a hierarchy of health professionals: "If it's drugs I go straight to the pharmacist. The doctor don't know shit... They don't get training in drugs basically". Zoe also articulated a hierarchy of preferred consultants that went from the health food shop, to the pharmacy, to the doctor as the last step.

Natasha was reluctant to take a remedy suggested by a friend because of concerns that it might interfere with her medications. To resolve this she would consult with her chiropractor who is "probably at the top of the hierarchy - the chiropractor... seems to know more about how systems work and how things affect each other". Sylvia consults "some pretty heavy reference books" in her bedroom but identifies networks of health professionals who use essential oils as important in ensuring that she is using them in a safe way.

Hazel explains why she took advice from her pharmacist about treatment for her heartburn which was a result of taking nurofen for period pain. Her doctor had suggested taking an additional medication to "mask the side effects of the acid", which Hazel thought was "a very dumb thing to do". But also Hazel's nutritionist had "more of an extreme view probably about diets and things like that and it would be a longer term approach - this really perfect diet all the time". Instead of these 'extremes' she went to the pharmacist who advised omega oils, which for Hazel was a middle way. Hazel works through her health advisors until she obtains the right advice for her. We see something similar with Jim. He rejects advice from Accident and Emergency staff to take medications for his rolled ankle, tries Arnica, and from his reading of "articles and stuff" he "heard that acupuncture was very good for injuries...so that's why I went for that and again I asked around and heard someone recommend a Chinese doctor that I went to. Again, I did my own research to check he's not one of these cowboys or anything like that”. Jim's strategy evolves in dynamic fashion as he draws on different sources.

Health professionals could also play a role in legitimating decisions. The following relates to a herbal based medication - Anti-Flamme - that Zoe uses: "I see that my physiotherapist uses it as well so that's given me a bit more confidence" as Zoe does not have so much faith in people who prescribe remedies. 
Relatives and friends are also sources of strategies. Hazel takes a zinc formula for colds that "my brother turned me on to". She also used grapefruit seed extract as she had had "a few funny years of poor health". She took it on the advice from a friend:

"She's a nurse, her husband's a doctor but they're also into alternative medicines themselves ... If you look on the Internet, in fact, with this it's not clear. It doesn't have lots of research and there's actually websites that say, 'Don't take this stuff, it's toxic'...I do think what triggered it was my friend suggesting it".

Hazel here considers different sources of information before following the advice of a friend with high health capital. Later she reflected on her participation in the research and concluded that "I think I listen to my friends a lot" and provided an example of following a friend's advice to use xylitol for her daughter's ear infection. Tania had a friend who is a naturopath and so is "immersed in that world as well". Avril and her partner "know quite a few people who are into some kind of healing or another", including acupuncturists, osteopaths and chiropractors. Sylvia had endometriosis that conventional approaches had not resolved and a friend said "look, I've heard this stuff's really good, why don't you go and have a chat to them?" referring to aromatherapy, to which Sylvia became a strong convert.

In a household with a tradition of using homeopathic remedies, we see a form of cultural or social capital at play around medication expertise with the household drawing on strong family networks. The following relates to a hypothetical discussion about sore throats, where Jason, the son, just asks mum for advice. Fleur, the mother, says "we'd be giving them some Echinacea straight away and then if it carried on then we might be going onto something else" and if required "we'd be ringing up my mother [who is a homeopath] and, 'What do we need for this kind of symptom and that kind of symptom?" In this instance the mother claims a particular level of expertise, but calls on her own mother's expertise in situations that are not so common. Tania took Psyllium hulls to promote bowel motions as suggested by her mother who was "diagnosed with cancer 38 years ago" and subsequently "went totally holistic". Orthodox prescriptions are also transferred across generations. Hazel takes Ibuprofen when menstrual pain stops her sleeping, something "my Mum told me about”. Similarly Sylvia used Bonjela teething get for her baby, stating it was "my Mum who told me about this". In this data men did not note intergenerational influences and fathers were not identified as important in health networks.

Workplaces could act as a source of therapeutic information that comes into the household. Jim notes that "at work you'll be sitting at the table and you'll find out this person has done this or found out and I'll always ask...'How did you find it? Have you used it for long?' and I just put that in my memory bank". Dan works at a child care facility. The consenting systems at the facility provoked researching activities: "In the first aid kits you have to sign for whether or not you're happy for your child to have these alternatives medicines used and having done some research on them following that decided that I was happy for them to be used and later found that actually they were quite effective". Roles and observations outside the household would 'come inside', for example, Jim in his role as an ambulance officer had "taken a lot of kids [in the ambulance] that had just had their immunisations" which "reinforced what I was doing was right", which was to avoid vaccinations. A workmate of Avril's was a yoga teacher and he "gave me that mixture and told me to take it. It's an Auyervedic [constitution] thing". Similarly Louisa used a homeopathic remedy recommended by a colleague at work who was a homeopath. She was "beside" herself as a result of frequent urinary tract infections that were 
not responding to specialist help from her urologist or others, but her colleague's recommendation "worked so well that I continue to use it". Workplaces are then sources of information, spaces of observation and can provoke research into wellness strategies that are deployed in the home.

As noted, householders undertake their own research in relation to wellness strategies. It is well established that people use the Internet to access information about medications (Nettleton 2004), and for participants in this research it was no different. The Internet can be used to access material from trusted sources and organisations, as with Sylvia who, in relation to the use of essential oils, states that "there's a couple of very reputable international websites...you can soon find people who are actually trained who aren't going to put you wrong”. The Internet can be used to try and locate specific treatment advice. Avril used the Internet to explore orthodox options for her jaw clenching and noted that besides a splint "they don't propose any alternatives". This gave her license to explore other options outside of dentistry. It was common for householders to use the Internet to check up on prescribed medications. Tania "was researching drugs for Mum. They wanted to get her on different things and I'd have a look up and print it up and Mum was, like, 'I ain't taking that shit”. Similarly Mark was prescribed a cholesterol-lowering medication "and when I read up about it I don't want to take it”.

The following, from a household where the mother questions medical advice at times, shows the complex connections made in decision making - the health professional, the Internet and her own sense-making determine the way in which she uses a prescribed medication. Janice avoids prescription medications but was put on Prednisone for "glue ear". At home she "looked it all up and looked at what the side effects could be...and thought, well, the pain is so bad I'll do what they say". Janice weighed up the different sources of information in relation to her condition before making a decision.

Householders demonstrated awareness of concerns about the Internet as a trusted source drawing on different rhetorics of reliability (Nettleton et al. 2005). Paula and Mark were impressed with what they heard on the radio about a medication for blood pressure. They checked it "on the computer" but would not purchase the product until they had consulted with "the blood doctor".

Ingrid undertook systematic research when she was newly diagnosed (30 years ago) with MS before the dominance of the Internet: "I went to the library and got every book I could on MS and then I started seeking people who had MS and also I started asking amongst all my friends for a suitable naturopath".

Louisa, who also has MS, found that links to the MS association and their material was important: "I read the latest research updates and there is a chap called George Jelinek, who is a trained emergency medicine physician who has MS and he's done a lot of research and he's researched all the research as well. So he's worked out what's good research, what's not good research and gone through and come up with a plan and fish oils was one of those".

Identifying wellness strategies could also be less systematic. Hazel took selenium because she "read an article that really influenced me years ago about how there's very little Selenium or none in the New Zealand soil". Reg, who uses a variety of supplements "Just picked [information] up here and there... some things come along and I read about it and either I like that idea or I can see the logic in that and adopted it". 
Deciding on what to pursue could involve time and effort and negotiation in the household. Janice stated that she liked "to know reasons for things" and gives the example of Losec prescribed for her 13 year old: "And I read about it and didn't like what it was what the side effects could be and the contraindications and so then I went back and thought, okay, well, he said these are the symptoms. Let's look at the symptoms and see what other things could be and what we can do about it".

It was rare for participants to claim influence from television. Natasha, however, suspected that she chose a particular cold sore medication because she "probably just saw it on the TV or something. Just marketing”. It was more common for participants to suggest that they were not influenced by that particular source.

In sum, friends and relatives can be important sources informing wellness strategies, and health professional advice is assessed for its merit and brought into the mix to determine the treatment plan in the household. We also see here the Internet being used in different ways, to find good advice, to check up on prescribed medications, to follow up on information gathered elsewhere and then assessed against other sources of information. Sources of advice are multiple and at times at variance. Householders make sense of this, hierarchizing in terms of trust and seeking out sources that fit with their own understandings and desires, using some sources to subvert the recommendations from others.

\section{Discussion}

Householders undertake a range of prescribing and diagnostic activities drawing on a range of resources. They deconstruct the worlds of scientific 'fact' (which for Latour is simply a stabilized proposition) and theoretical purity by adding and subtracting from sources, varying recommendations and combining different approaches. The health expertise found in consulting rooms, Internet sites and pharmacies is decentred as householders develop their own hierarchies of sources based on combinations of experience, worldview and the particular issue they are trying to resolve and how that issue responds to their attempts at resolution. Using the concept of hybrids in a Latourian sense and taking as central de Certeau's perspective on the productive capacities of everyday life, householders should not be seen as passive consumers of dominant discourses but are active producers of hybridised medication practices beyond the purview of medicine's centres of calculation.

It is uncommon for households to provide accounts of just doing what the doctor tells them. Householders generally construct themselves as actively participating in disputes over risks and confronting uncertainties in determining their wellness strategies. Hobson-West notes this in relation to what she calls vaccine-critical groups, where those who question the use of vaccines on their children feel required to take on greater responsibility for their children's health and understand complex arguments about immunisation (Hobson-West 2007). Householders similarly undertake this sort of work and responsibility, to varying degrees, in relation to their therapeutic practices. The exploration of household use of medications suggests that there is a continuum here in relation to a wide array of medication practices, with most householders not relying on just one source of advice.

Viewing households as hybrid centres of medication practices has implications for a sociological understanding of households and health and for health practitioners' conceptions of the consultation. 
In terms of sociological understandings of households, Prior has argued that we should be cautious about imputing 'expertise' to those who have not been medically trained as they can be wrong and they only have their own experience or the experience of others close by to draw on. He states that patients are "rarely skilled in matters of (medical) fact gathering, or in the business of diagnosis" and are not "skilled and practised in the diagnosis and management of illness" (Prior 2003). The skill levels of householders is not the subject of this paper - but the ubiquitous nature of 'fact' and opinion gathering from a wide range of sources and the requirements to make diagnoses to determine such decisions as when to call on expertise are quotidian. This is not to deny that householders identify outside experts who can, and often do, play a central part in their determinations. Bissell and colleagues have argued, in relation to the use of non-prescription medicines, that lay people "engender a sense of agency or lay expertise over the body and its maintenance" whilst remaining dependent on medicine (Bissell et al. 2001).But as McClean and Shaw demonstrate in relation to food risks and alternative medicine use, laypeople "adopt, mimic, critique, or rewrite expert positions" (McClean and Shaw 2005). The adopting and rewriting occurs not only in relation to experts, but also to friends, relatives, colleagues and a range of searching activities. However, we should note a limitation here in that this research did not focus on acute conditions, which would likely shift the focus of trust onto a narrower range of influences.

It is argued here that households are a central site of health practices and decision-making. Historians have claimed that home health practices became severely delimited with the rise in dominance of the medical profession (Risse 1993; Wear 1995). In seventeenth century England "Well-stocked homes had kitchen-physic: bottles of homebrewed or shop-bought purges, vomits, pain-killers, cordials, febrifuges (medicines to reduce fever) and the like" and people were "less doctor dependent than today" (Porter 1987: 29). Anthropologists have shown how home health practices are predominant in non-western cultures where the purchase of 'professional biomedicine' is limited, implying a lack of home health practices in the west (Waldstein 2010). Sociologists have argued that in recent times a new medical cosmology has developed as a consequence of medical knowledge becoming unbound from medical institutions through new technologies, primarily through the use of the Internet (Nettleton 2004). The research discussed here suggests that the disappearance of therapeutic practices from the home in the west has been overstated and overlooked. Professional biomedicine does have a purchase on the home in contemporary times - but the practices of professional biomedicine are thoroughly mixed with other wellness strategies and re-worked in the home. The research also suggests that although the Internet can be a source of wellness strategies there are, and always have been, many other sources going across generations, workplaces and through social networks as well as technological networks. By looking at the wellness strategies in the home it can be argued that medical knowledge has never been successfully bound up within medical institutions or professional biomedicine. Medical practitioners and other health providers have been but one source of strategies.

This view of households as the space where decisions are made based on outside advice, experimentation and observation has consequences for health practitioners and the concepts they use to enhance clinical decision making and patient compliance. For example, the meaningfulness of the goal of 'concordance' in medical decision-making that became a prominent feature of consultation discussions in the 1990s (Mead and Bower 2000) is questioned. Concordance has been defined as: 
An agreement reached after negotiation between a patient and a healthcare professional that respects the beliefs and wishes of the patient in determining whether, when, and how medicines are to be taken (Dickinson et al. 1999).

Such a notion has an underlying assumption of the patient as individualised and decisionmaking as a static event that occurs once in a consulting room - as opposed to decision-making being a dynamic and embedded within a networked collective - the medical doctor being but one (although very often crucially important) node in the network. As such this view challenges the centrality of the medical consultation in medication practices and directs us to the everyday and collective nature of health-care decision-making.

\section{Conclusion}

This paper has drawn on the work of Latour to argue that households can usefully be seen as hybrid centres of therapeutic practice. The use of the concept of hybrid provides a focus that questions the compartmentalizing of categories into orthodox and alternative, artificial and natural. These are intertwined and mixed in health maintenance activities in the household. The term hybrid also supports the image of households as nodes in complex networks of advice, practice and expertise. Drawing on de Certeau it is argued that households are active producers of medication practices, using but manipulating frameworks drawn from outside the household. This manipulation and production of practices applies as much to the frameworks of alternative practitioners as it does to the frameworks of orthodox medicine.

Householders necessarily develop expertise in wellness strategies through experimentation, research activities and consultations with numerous advisors and acquaintances. They draw on their own hierarchies of trust, they use health advice as tools for developing their own medication practices and wellness strategies, and, following de Certeau, householders subvert normative frameworks and evade disciplinary practices. Subversion can be seen in processes of rejecting, modifying and tactically forgetting. Personal medication practices develop from practical concerns over medication use, resource issues, congruence with worldviews and through processes of experimentation and observation. Sociologists and professional health advisors can usefully conceptualise the household as the centre of a networked therapeutic practice that is actively hybridising therapeutic advice.

\section{Acknowledgements}

This research was supported by grants from the Health Research Council of New Zealand and the Marsden Fund of the Royal Society of New Zealand. We would like to thank Linda Nikora and Helen Madden for their input into research design and data collection and Kathryn McGuigan for assistance with data collection.

\section{Endnotes}

1. In relation to prescription medications New Zealand has a public health system where approved drugs are provided under Government subsidy. General Practitioners are primarily responsible for prescribing medications with most GPs operating under fee-for-service arrangements with some government subsidy for these consultations. 
Table 1: Sample characteristics

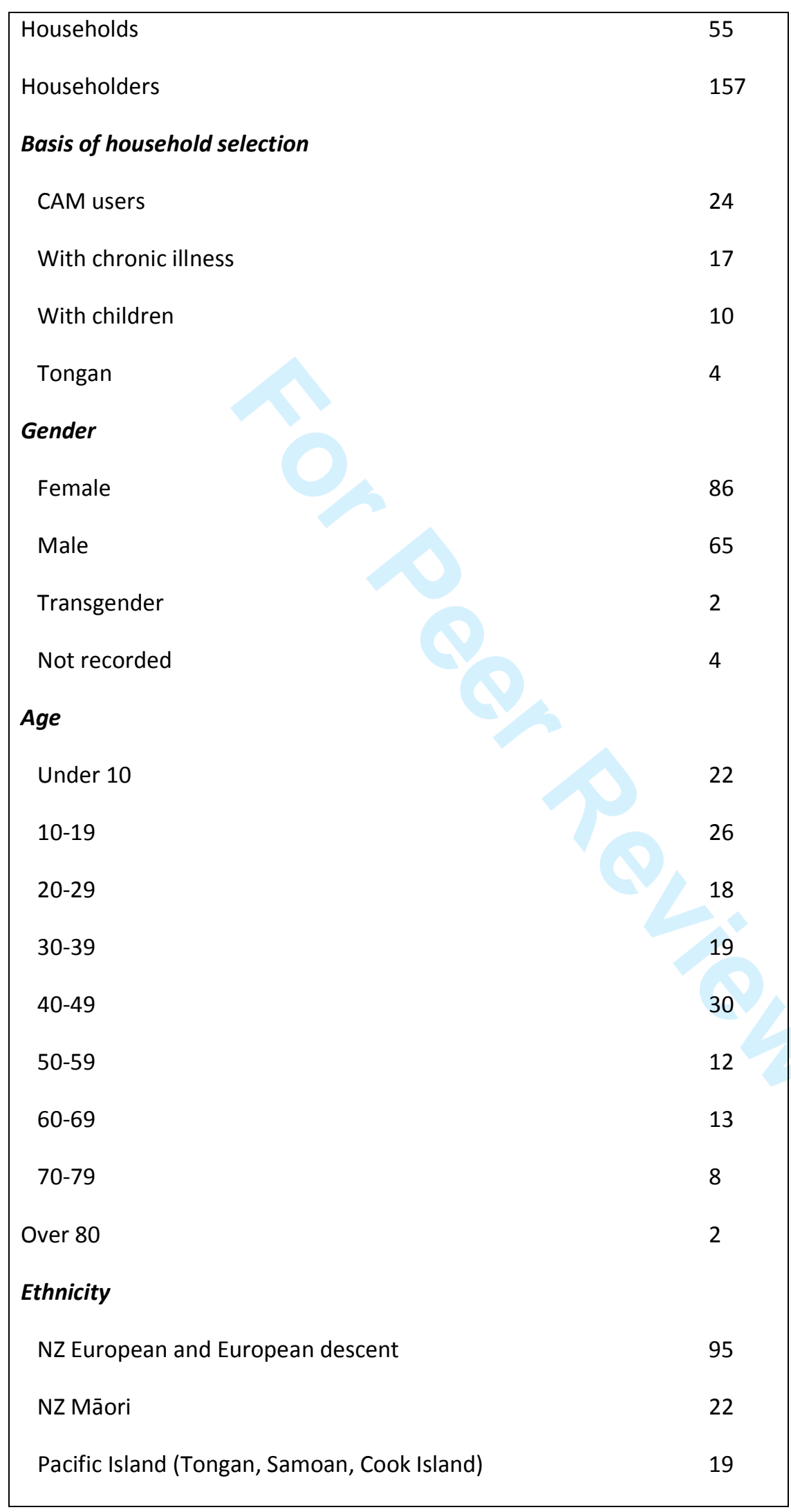


1

2

3

4

5

6

7

8

9

10

11

12

13

14

15

16

17

18

19

20

21

22

23

24

25

26

27

28

29

30

31

32

33

34

35

36

37

38

39

40

41

42

43

44

45

46

47

48

49

50

51

52

53

54

55

56

57

58

59

60
Chinese and Chinese descent 13

Other (Asian and Middle Eastern)

2

Not recorded 
References

Bissell, P., Ward, P. and Noyce, P. (2001) The dependent consumer: Reflections on accounts of the risks of non-prescription medicines, Health, 5, 1, 5-30.

Blok, A. and Jensen, T. (2011) Bruno Latour: Hyrbid thoughts in a hybrid world, London and New York: Routledge.

Buchanan, I. (2000) Introduction to part III, in G. Ward (ed), The Certeau reader. Oxford: Blackwell, pp. 97-100.

de Certeau, M. (1984) The practice of everyday life, Berkeley: University of California Press.

Dew, K. (2001) Modes of practice and models of science in medicine, Health: An Interdisciplinary Journal for the Study of Health, Illness and Medicine, 5, 1, 93-111.

Dickinson, D., Wilkie, P. and Harris, M. (1999) Taking medicines: concordance is not compliance, British Medical Journal, 319, 787.

Hobson-West, P. (2007) 'Trusting Blindly Can Be the Biggest Risk of All': Organised Resistance to Childhood Vaccination in the UK., Sociology of Health and IIIness, 29, 2, 198-215.

Jutel, A. (2011) Putting a name to it: Diagnosis in contemporary society, Baltimore: The John Hopkins University Press.

Latour, B. (1988) The politics of explanation: an alternative, in S. Woolgar (ed), Knowledge and reflexivity: new frontiers in the sociology of knowledge. London: Sage, pp. 154-176.

(1992a) The costly ghastly kitchen, in A. Cunningham and P. Williams (eds), The laboratory revolution in medicine. Cambridge: Cambridge University Press, pp. 295-303.

(1992b) Where are the missing masses? The sociology of a few mundane artefacts, in W. Bijker and J. Law (eds), Shaping Technology/Building Society: Studies in Sociotechnical Change. Massachusetts: MIT Press.

(1993) We have never been modern, Cambridge, Mass.: Harvard University Press.

(2005) Reassembling the Social: An Introduction to Actor-Network-Theory, New York: Oxford.

McClean, S. and Shaw, A. (2005) From schism to continuum? The problematic relationship between expert and lay knowledge - An exploratory conceptual synthesis of two qualitative studies, Qualitative Health Research, 15, 6, 729-749.

Mead, N. and Bower, P. (2000) Patient-centredness: a conceptual framework and review of the empirical literature, Social Science \& Medicine, 51, 1087-1110.

Nettleton, S. (2004) The emergence of e-scaped medicine?, Sociology, 38, 4, 661-679.

Nettleton, S., Burrows, R. and O'Malley, L. (2005) The mundane realities of the everyday lay use of the internet for health, and their consequences for media convergence, Sociology of Health \& IIIness, 27, 7, 972-992.

Porter, R. (1987) Disease, medicine and society in England 1550-1860, Basingstoke: Macmillan.

Prior, L. (2003) Belief, knowledge and expertise: the emergence of the lay expert in medical sociology, Sociology of Health \& IIIness, 25, 41-57.

Risse, G. (1993) Medical care, in W.F. Bynum and R. Porter (eds), Companion encyclopedia of the history of medicine. London \& New York: Routledge, pp. 45-77.

Tilley, C. (2006) Introduction: Identity, place, landscape and heritage, Journal of Material Culture, 11, 7-32.

Waldstein, A. (2010) Popular medicine and self-care in a Mexican migrant community: Toward an explanation of an epidemiological paradox, Medical Anthropology, 29, 1, 71-107.

Ward, G. (2000) Introduction, in G. Ward (ed), The Certeau reader. Oxford: Blackwell, pp. 1-14.

Wear, A. (1995) Medicine in early modern Europe, 1500-1700, in L.I. Conrad (ed), The western medical tradition: 800 BC to AD 1800. Cambridge: Cambridge University Press, pp. 215-361.

Williams, C. (2000) Alert assistants in managing chronic illness: the case of mothers and teenage sons, Sociology of Health \& IIlness, 22, 2, 254-272. 
1

2

3

4

5

6

7

8

9

10

11

12

13

14

15

16

17

18

19

20

21

22

23

24

25

26

27

28

29

30

31

32

33

34

35

36

37

38

39

40

41

42

43

44

45

46

47

48

49

50

51

52

53

54

55

56

57

58

59

60
Williams, G. and Popay, J. (1994) Lay knowledge and the privilege of experience, in J. Gabe, D. Kelleher and G. Williams (eds), Challenging medicine. London: Routledge, pp. 118-139. 